\title{
Automated vision occlusion-timing instrument for perception-action research
}

\author{
John Brenton ${ }^{1} \cdot$ Sean Müller ${ }^{1} \cdot$ Robbie Rhodes $^{2} \cdot$ Brad Finch $^{2}$
}

Published online: 23 February 2017

(C) Psychonomic Society, Inc. 2017

\begin{abstract}
Vision occlusion spectacles are a highly valuable instrument for visual-perception-action research in a variety of disciplines. In sports, occlusion spectacles have enabled invaluable knowledge to be obtained about the superior capability of experts to use visual information to guide actions within in-situ settings. Triggering the spectacles to occlude a performer's vision at a precise time in an opponent's action or object flight has been problematic, due to experimenter error in using a manual buttonpress approach. This article describes a new laser curtain wireless trigger for vision occlusion spectacles that is portable and fast in terms of its transmission time. The laser curtain can be positioned in a variety of orientations to accept a motion trigger, such as a cricket bowler's arm that distorts the lasers, which then activates a wireless signal for the occlusion spectacles to change from transparent to opaque, which occurs in only $8 \mathrm{~ms}$. Results are reported from calculations done in an electronics laboratory, as well as from tests in a performance laboratory with a cricket bowler and a baseball pitcher, which verified this short time delay before vision occlusion. In addition, our results show that occlusion consistently occurred when it was intended - that is, near ball release and during mid-ball-flight. Only $8 \%$ of the collected data trials were unusable. The laser curtain improves upon the limitations of existing vision occlusion spectacle triggers, indicating that it is a valuable instrument for perception-action research in a variety of disciplines.
\end{abstract}

Sean Müller

s.muller@murdoch.edu.au

1 School of Psychology and Exercise Science, Murdoch University, 90 South Street, Murdoch, Perth 6150, Australia

2 B3 Electronic Design Pty Ltd, Perth, Australia
Keywords Vision occlusion spectacles · Automatic trigger · Laser curtain · Wireless

Milgram's (1987) invention of spectacles that can occlude the availability of visual information to an observer has been invaluable to researchers in different disciplines involved in visual-perception-action research. Vision occlusion spectacles have been used to understand the time course of visual information that guides motor skills such as reaching to grasp an object (Whitwell, Lambert, \& Goodale, 2008), interception of a fast-moving object (Marinovic, Plooy, \& Tresilian, 2009), and maintenance of balance in movement disorders (Morris et al., 2015). Over the past 20 years, an increasing number of studies have used vision occlusion spectacles to investigate the time course of visual information that guides complex, whole-body, high-speed sports skills such as return of serve in tennis and cricket batting. Vision occlusion spectacles are popular with researchers because they allow the availability of visual information to be manipulated within in-situ settings and coupled to complex actions such as movements of the whole body to strike a high-speed object such as a tennis ball.

Starkes, Edwards, Dissanayake, and Dunn (1995) were the first to use vision occlusion spectacles to investigate the time course of visual information pickup for anticipation in the high-speed sports skill of serving in volleyball. Skilled and novice volleyball players stood on a court and wore the spectacles, which were connected by a cable to a timer on the sideline. Participants observed opponents deliver different types of serves that were occluded (spectacles opaque) at different time points in the server's action, and they had to make a nonmotor prediction of the serve's landing position by placing a marker on the court. The experimenter manually triggered the spectacles to occlude the participant's vision of the server's action by simultaneously watching the server's action unfold and 
pressing the button on the timer to correspond with preplanned kinematic events. Occlusion was targeted at events prior to, at, and after the server's ball-hand contact. The authors reported that the time delay for spectacles to occlude using a cable interface was $2 \mathrm{~ms}$. However, due to error in the timing of manual triggering (likely due to reaction time delays), a high-speed camera was used to film the server's action and record the created vision occlusion conditions. The footage was sorted post-hoc to confirm which trials could be retained for data analysis on the basis of preplanned temporal-occlusion conditions. The findings indicated that skilled university players could predict serve landing locations with less error than could novices. Their study spawned several others within in-situ settings that have used a manually triggered and post-hoc trial-sorted approach to demonstrate that experts have anticipation skills superior to those of less skilled players in modified squash game scenarios (Abernethy, Gill, Parks, \& Packer, 2001), tennis return of serve (Farrow \& Abernethy, 2002, 2003; Farrow, Abernethy, \& Jackson, 2005), cricket batting (Müller \& Abernethy, 2006; Müller et al., 2009), and baseball batting (Müller, Lalović, Dempsey, Rosalie, \& Harbaugh, 2014).

Although manual triggering of vision occlusion spectacles and post-hoc sorting of captured trials has allowed advancements to be made in terms of in-situ knowledge of expert visual-perceptual-motor skill, there are also some limitations. First, the cable connection from the vision occlusion spectacles to the manual trigger timer may restrict the broad range of movements of performers in sports such as cricket or tennis that requires lower body positioning and bat-ball interception. Accordingly, a cable can impede the perception-action coupling of the motor skill that exists in the actual game setting. Recently, through use of an automated force plate trigger and a cable connection to vision occlusion spectacles, it was reported that a tethered system does not impede the natural striking pattern of cricket batsmen (Mann, Abernethy, \& Farrow, 2010; Mann, Abernethy, Farrow, Davis, \& Spratford, 2010). However, these studies used a smaller range of cricket batting striking patterns. Burroughs (1984) had previously developed a helmet on which an opaque visor was triggered to occlude a baseball batters vision by the pitcher's foot landing on a pressure-sensitive pad. However, specific details such as whether the system was tethered and the time delay until occlusion were not reported clearly. Second, high-speed sports skills such as the cricket bowler's delivery action and ball flight occurs under $1 \mathrm{~s}$. Delays due to the reaction time of the experimenter in depressing the timer button to correspond with a simultaneously observed bowler's kinematic event, as well as transmission of the signal to switch the spectacles from transparent to opaque, can be problematic in terms of the precise occlusion timing relative to a kinematic event. For example, manual triggering incurs a range of occlusion timing that is approximately 200-300 ms (see, e.g., Farrow \& Abernethy, 2003; Müller, Brenton, Dempsey, Harbaugh, \&
Reid, 2015). Again, by means of an automated force plate trigger system, it has been reported that a cricket batsman's vision can be temporally occluded within a 50-ms range prior to or after ball release of a cricket bowler's action (Mann, Abernethy, Farrow, et al., 2010). Third, the delay due to use of a manual trigger of occlusion can cause loss of data because the timing of occlusion does not correspond with the preplanned kinematics event(s). For example, it may be necessary to omit approximately $30 \%$ of the total completed trials per participant due to incorrect triggering of occlusion timing in relation to the corresponding kinematic event (see, e.g., Müller et al., 2015). Loss of data has been reduced below this percentage using an automated occlusion system (Mann, Abernethy, Farrow, et al., 2010).

Although attempts have been made to automate occlusion timing and minimize the limitations mentioned that are associated with manual triggering, further advancements to occlusion-timing technology are necessary. As was mentioned by Starkes et al. (1995), vision occlusion technology needs to be transportable so that researchers can assess participants visual-perceptual-motor skill in a variety of in-situ settings, such as a performance laboratory, actual game performance venue, or indoor venue that can protect expensive equipment from the weather. Vision occlusion spectacles also need to have wireless triggers in order to accommodate the broad range of sports skill movement patterns, such as forward and backward foot movements, or ball interception with vertical and horizontal bat swings in dynamic and complex skills such as cricket batting. Furthermore, it would be beneficial to consistently occlude vision at specific, preplanned kinematic events.

This article outlines an instrument that is capable of making these advances in terms of an automated wireless trigger of vision occlusion spectacles. The purpose of this article is to (a) outline a new automated wireless trigger for vision occlusion spectacles, (b) present data on the time delay for automated wireless triggers of vision occlusion spectacles from transparent to opaque, and (c) present data on automated wireless vision occlusion timing in relation to two exemplar highspeed sports skills: cricket bowling and baseball pitching.

\section{Method}

\section{Participants}

Two skilled male players participated in this study; one was a baseball pitcher, and the other was a cricket bowler. Both had played club-level baseball and cricket, respectively. The participants were recruited in order to obtain occlusion timing data for baseball pitching and cricket bowling kinematic events, which are common motor skills that have been studied in the in-situ temporal-occlusion literature. 


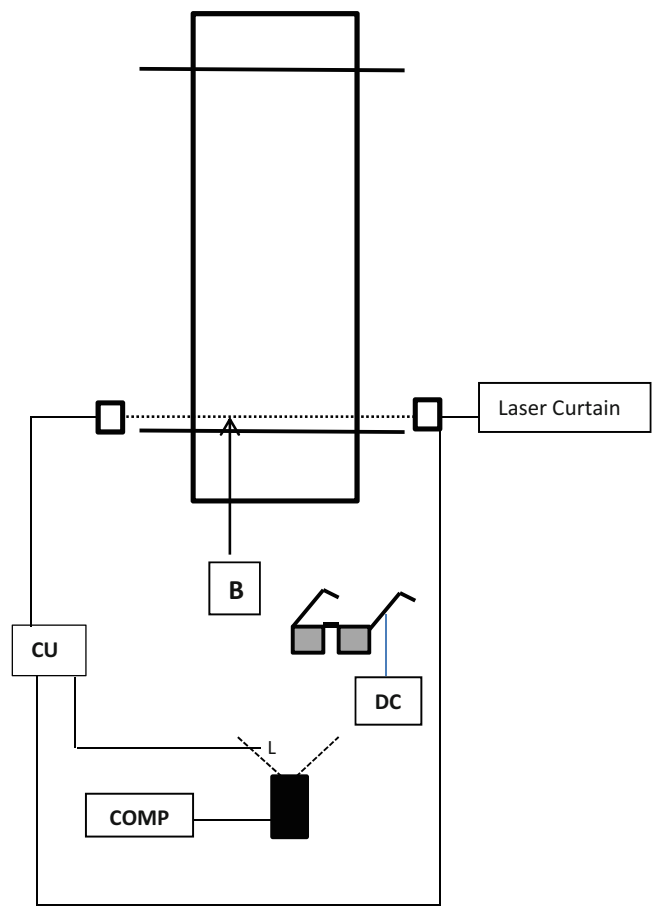

Fig. 1 Schematic diagram of the instrumental setup for one of the tests that was conducted with a cricket bowler. Vertical laser tower pairs were placed on stands on either side of a cricket pitch to create a laser curtain near the point where the bowler would release the ball. The bowler (B) ran for approximately $5 \mathrm{~m}$ and released the ball at the position of the laser curtain. The control unit (CU) was connected to the laser curtain and light-emitting diode (L) by cables (indicated by solid lines). The CU communicated wirelessly with the driver controller (DC), which was connected by cable to the vision occlusion spectacles. A high-speed camera (solid black square) was controlled by a laptop computer (COMP) to capture the bowler's delivery action at the position of the light curtain, as well as the illumination of the light-emitting diode

\section{Instruments}

Figure 1 provides a diagrammatic representation of the instrumental setup for automated vision occlusion timing relative to kinematic events in baseball pitching and cricket bowling. The new automated occlusion-timing instrument consists of (i) a laser curtain wireless device capable of triggering the vision occlusion spectacles in response to a kinematic event, such as the pitcher's or bowler's arm distorting part of the laser curtain; (ii) a control unit that operates the whole system; and (iii) a driver controller that is connected to the vision occlusion spectacles' driver.

The laser curtain consists of a transmitter and a receiver tower pair that is made of an alloy frame to protect the lasers housed inside. The transmitter tower consists of $32.5-\mathrm{mW}$ lasers, with the tower tethered by a direct current (DC) cable to a control unit. The receiver tower consists of 32 phototransistors that detect the light from each of the corresponding transmitter tower lasers. The receiver tower is also tethered to the control unit through a standard network cable (RJ45 connector). The towers can be set up in any orientation,

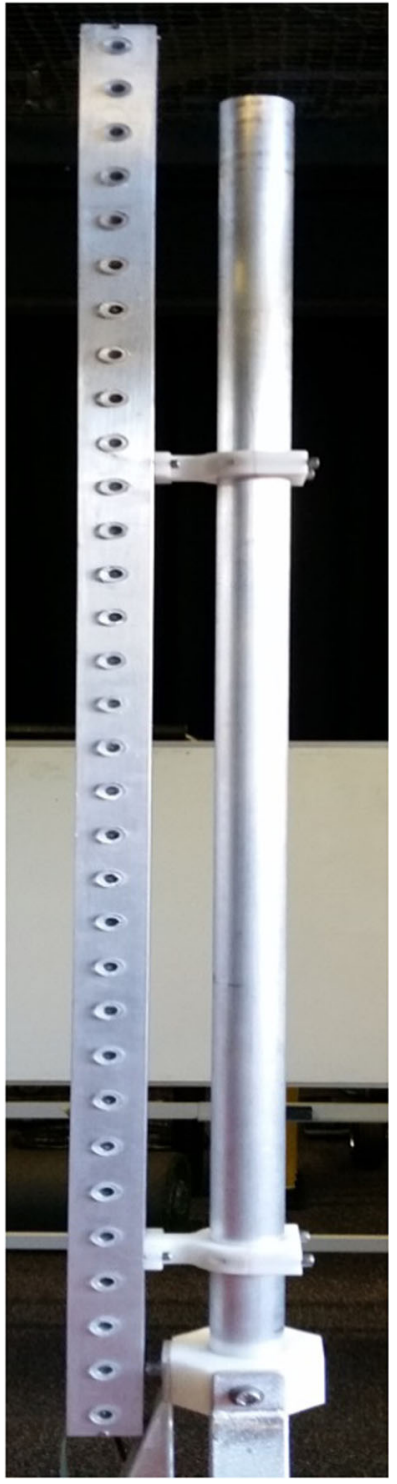

Fig. 2 Transmitter tower lasers mounted vertically on a stand

but for the test in this article, they were mounted vertically on stands approximately $3 \mathrm{~m}$ apart so that the bowler/pitcher could perform their movements in-between the towers, distort the laser curtain, and trigger the spectacles to occlude vision either near ball release or during ball flight (see Fig. 2).

The control unit is used to power both towers, for instantaneous communication between the towers, and to specify the timing of occlusion relative to a kinematic event (see Fig. 3). In turn, the control unit can be powered by a $12-\mathrm{V}$ supply (four AA batteries) and can operate five tower pairs if needed. The top panel of the control unit includes switches that can be positioned at Stage 1 or 2 to selectively operate specific tower pairs. The center dials are used to select the mode of operation, such as to align the lasers and use specific tower pairs (or stages) to create occlusion or no occlusion (in the latter case, the lasers turn on but do not trigger vision 


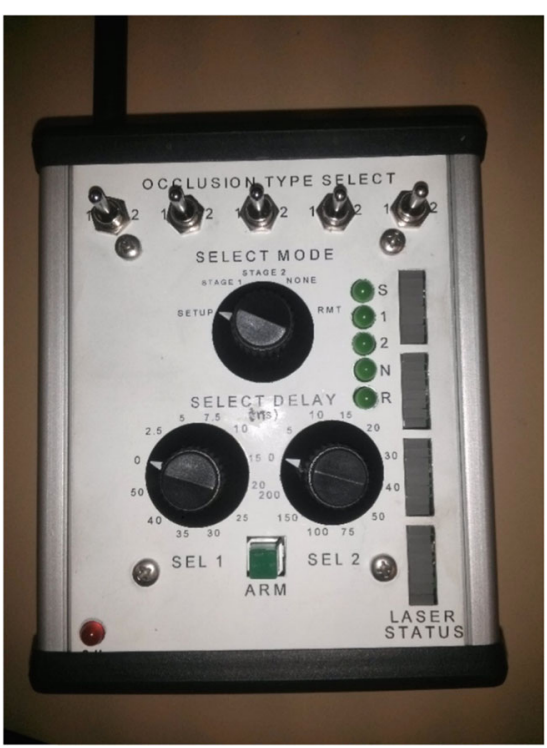

Fig. 3 The unit that controls the laser curtain, light-emitting diode, and wireless driver controller (connected to the spectacles driver)

occlusion). The lower dials are used to select time delays from the instant a kinematic event, such as a bowling arm, distorts part of the laser curtain. For example, the delay can be set to $0 \mathrm{~ms}$ if the intention is to create temporal occlusion near the point of a kinematic event such as ball release. Alternatively, if the intention is to create temporal occlusion after a kinematic event such as ball release, then a delay (e.g., $200 \mathrm{~ms}$ ) can be set up on the dial. The "arm" button is used to prepare the tower pairs to accept a kinematic event, such as a cricket bowler's arm distorting part of the laser curtain, to trigger the occlusion spectacles. The light-emitting diode (LED) on the left-hand side of the control unit flashes when the tower pairs are armed, is on when the spectacles are transparent, and is off when the spectacles are opaque. Thirty-two dual-colored LEDs on the right-hand side of the control unit are illuminated during setup to confirm laser alignment. A separate cable is connected at one end to the control unit, and at the other end its LED is positioned in the high-speed camera's field of view to signal the time when the occlusion spectacles become opaque.

The driver controller is connected to the vision occlusion spectacles' driver through a short RJ11 lead (see Fig. 4). The controller communicates wirelessly with the control unit at $434 \mathrm{MHz}$, modulated by an $800-\mu$ s square wave when it is idle, changing to a $300-\mu$ s modulation to signal occlusion. The driver controller is powered by six AAA batteries housed in a case and connected by a short cable to the driver controller. The driver controller, spectacle driver, and battery power supply are housed within a portable bag that is usually strapped to the participant (e.g., batter), with the vision occlusion spectacles being connected to the driver, as had been done in previous in-situ temporal occlusion studies (see, e.g., Müller et al., 2015).

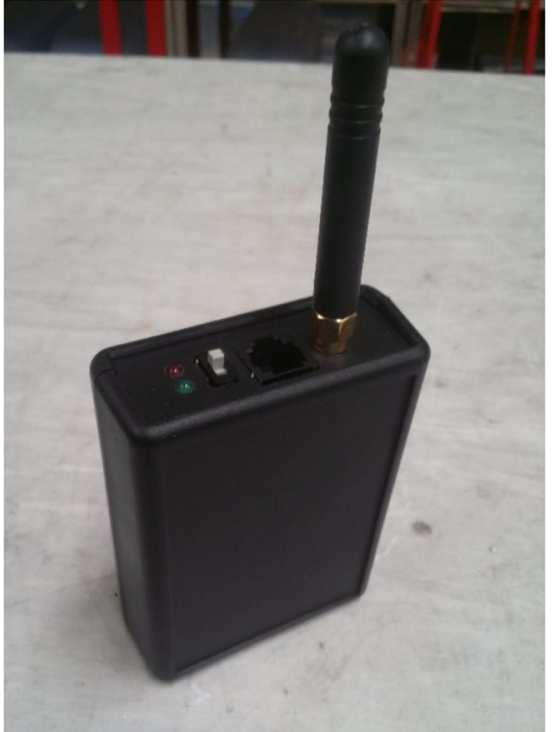

Fig. 4 The driver controller that is attached to the spectacles driver and controls the vision occlusion spectacles through wireless communication with the control unit

A high-speed camera that sampled up to 1,000 frames per second was used to capture kinematic events such as ball release and ball flight, LED illumination, and in the foreground the occlusion spectacles. This allowed the vision occlusion time to be quantified using a frame-by-frame analysis at 250 and 1,000 frames per second.

\section{Procedure}

First, in an electronics laboratory, the experimenters calculated the time from when the laser curtain was distorted to the moment the vision occlusion spectacles became opaque. Second, in a performance laboratory, one of the experimenters distorted the light curtain with a hand for 18 trials, while the high-speed camera sampling at 1,000 frames per second filmed through the lens of the vision occlusion spectacles, in order to determine the time at which the LED was illuminated and the spectacles changed from transparent to opaque. Third, a baseball pitcher threw 60 pitches that consisted of two pitch types (fastball and curveball), while the pitcher's throwing arm distorted the laser curtain near ball release. Temporal occlusion was targeted near ball release for 30 trials using a 0 -ms delay, and in the remaining 30 trials occlusion was targeted during ball flight using a 200-ms delay. Fourth, a cricket bowler, using a short run-up, bowled 28 balls that consisted of two ball types (balls that landed farther away from and closer to the bowler), where, again, the bowler's hand distorted the laser curtain near ball release. For 15 of the balls, temporal occlusion was targeted near ball release using a 0 -ms delay, and for the remaining 13 balls, occlusion was targeted during ball flight using a 200-ms delay. Both the 
pitcher and bowler were filmed by a high-speed camera sampling at 250 frames per second.

\section{Results}

The time delay from when the laser curtain was distorted until the vision occlusion spectacles changed to opaque was $8 \mathrm{~ms}$, as calculated in the electronics laboratory. Figure 5 provides a diagram of the instruments and their component time delays, as calculated in the electronics laboratory. The diagram indicates that, within the total delay time for the vision occlusion spectacles to be switched from transparent to opaque, the majority of the time delay was due to the driver controller, driver, and the vision occlusion spectacles. All 18 trials captured in the performance laboratory consistently resulted in a time delay from when the laser curtain was distorted until the vision occlusion spectacles became opaque of $8 \mathrm{~ms}$ (at a filmed rate of 1,000 frames per second). In relation to the baseball pitcher, in the 30 trials during which temporal occlusion was targeted near ball release, occlusion occurred $12 \mathrm{~ms}$ after ball release (at the 250-frame-per-second filmed rate). In the remaining 30 trials, during which temporal occlusion was targeted $200 \mathrm{~ms}$ after ball release, occlusion occurred $212 \mathrm{~ms}$ after ball release (again at the filmed rate of 250 frames per second). In only one trial in the ball flight temporal-occlusion condition did the glasses not occlude at $212 \mathrm{~ms}$. In relation to the cricket bowler, for the 15 trials during which temporal occlusion was targeted near ball release, in ten of the trials we recorded occlusion occurring $12 \mathrm{~ms}$ after ball release (at the filmed rate of 250 frames per second), with occlusion occurring in the other four trials much earlier than ball release. In the remaining 13 trials, during which temporal occlusion was targeted during ball flight, in 12 of the trials we recorded occlusion occurring $212 \mathrm{~ms}$ after ball release (at the filmed rate of 250 frames per second), with occlusion on the other trial occurring much later.

\section{Discussion}

In this article, we initially outlined how vision occlusion spectacle technology has been used in several disciplines to investigate the time course of visual information that guides action. Thereafter, the article described a new automated laser light curtain technology that could wirelessly trigger vision occlusion spectacles to switch from transparent to opaque in a very short time. Quantitative evidence of the time delay for the spectacles to occlude (become opaque) was provided on the basis of calculations conducted in an electronics laboratory and by filming the spectacles change state at a very high frame rate. In addition, the time delay until occlusion was tested using two striking sports skillsbaseball pitching and cricket bowling-that are commonly used within in-situ occlusion studies. Collectively, significant advances have been made in automating and wirelessly triggering vision occlusion spectacles, which will benefit future research.

The new automated wireless laser curtain trigger for vision occlusion spectacles includes only one main piece of physical equipment in addition to what is commonly used in existing manual trigger systems. Even with recent advances in the automated occlusion of spectacles, a force plate that is not portable is still required; although a pressure pad system has been suggested as an alternative (Mann, Abernethy, Farrow, et al., 2010), the function of the pressure pad system has yet to be properly demonstrated with motor skills such as those used in cricket and baseball. In their seminal article, Starkes et al. (1995) identified the importance of portable vision occlusion spectacle technology, and in line with this vision, they used very little equipment to create and verify the temporal-occlusion conditions. The laser light curtain reported in this article could certainly be used on a volleyball court to measure anticipation of the serve, as in Starkes et al.'s study, but providing the participant with freedom of movement, due to the wireless trigger for the spectacles. The light curtain could also be used in other indoor sports venues - such as for cricket batting, baseball batting, tennis return of serve, and soccer goalkeeping, to name a few-which will protect the equipment from weather.

The results from this article demonstrate that when the new laser curtain is triggered by a kinematic event it can through wireless communication consistently switch the state of the occlusion spectacles from transparent to opaque in some $8 \mathrm{~ms}$. Previous studies have reported wireless trigger delays between 10-20 ms using instrument sampling rates of $200 \mathrm{~Hz}$ (see, e.g., Farrow \& Abernethy, 2003; van Soest et al., 2010). Using a much higher sampling rate of 1,000 frames per second in this article, it was possible to clarify that the actual time delay for the wireless vision occlusion spectacles to be switched to opaque is shorter than previously thought. The 8-ms wireless delay reported in this article is more than reasonable when considered in relation to the seminal in-situ occlusion study by Starkes et al. (1995) that used a cable and reported a delay of $2 \mathrm{~ms}$, as well as the seminal occlusion spectacles instrument article by Milgram (1987) that reported a delay of $4 \mathrm{~ms}$.

The automated laser curtain trigger reduced the range over which vision occlusion is created on the basis of preplanned kinematic events. In this article, the majority of the test trials resulted in vision occlusion consistently 


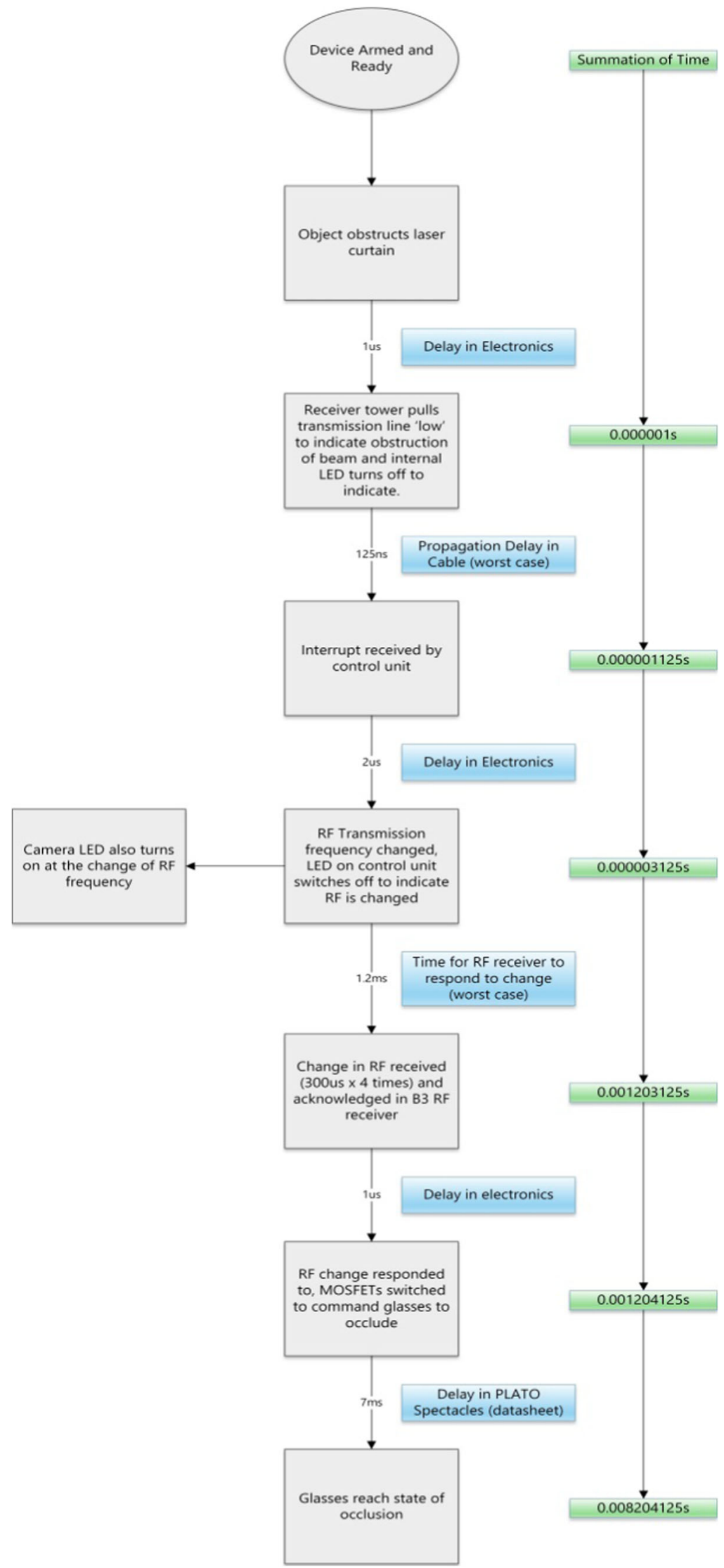

Fig. 5 Cumulative instrument time delays for the laser curtain, control unit, light-emitting diode, driver controller, and vision occlusion spectacles 
occurring $12 \mathrm{~ms}$ after ball release (using a 0 -ms set occlusion delay on the control unit) or $212 \mathrm{~ms}$ after ball release (using a 200-ms set occlusion delay on the control unit). This is a much smaller range of timings for creating occlusion than has previously existed in the literature for either manual triggering, at around $200 \mathrm{~ms}$ (see, e.g., Müller et al., 2015), or automated triggering, at $50 \mathrm{~ms}$ (Mann, Abernethy, Farrow, et al., 2010). It may be argued that for the ball release kinematic event, temporal occlusion in this article was created after ball release, which can be considered different from the pre-ball-release temporal occlusion conditions in several previous in-situ studies (e.g., Farrow \& Abernethy, 2003; Mann, Abernethy, \& Farrow, 2010; Müller \& Abernethy, 2006; Starkes et al., 1995). However, the broader range of temporal-occlusion timings prior to a key kinematic event such as ball release or racquetball contact in these previous studies needs to be taken into consideration. Accordingly, previous studies would not have consistently allowed the observer (e.g., a cricket batter) to view the final position of the distal limb segment such as the ball and hand at the point of ball release, which has been reported to be informative for expert anticipation (Loffing \& Hagemann, 2014; Müller, Abernethy, \& Farrow, 2006). Therefore, to be able to create temporal occlusion just after ball release appears to be important in order to fully understand the capability of performers to use complete key kinematic anticipatory visual cues.

The automated laser curtain was highly successful in preventing the significant loss of test trial data that commonly occurs when using manual triggering. In this article, of the total 88 test trials that were completed between the pitcher and bowler, on only seven trials was vision occlusion not created as intended, which is an $8 \%$ exclusion rate from the total completed test trials. This is considerably better than a manual wireless trigger method, which can incur a loss of approximately $30 \%$ of the total test trials. The significantly increased retention of the number of test trials that were completed would contribute to keeping trial repeats included in the test trial matrix during the planning phase of occlusion studies (to safeguard against data loss) to a minimum. Increased retention of trials can also minimize the time required for participants to complete in-situ studies, particularly since professional athletes are time-limited and often have workload restrictions relating to balls bowled or pitches thrown.

\section{Summary}

Details were presented here of a new automated wireless trigger for vision occlusion spectacles that includes a laser curtain, driver controller, and control unit. These instruments were checked in a series of tests with vision occlusion spectacles and a high-speed camera in both electronics and performance laboratories. Consistently, very small time delays were found with the electronic measures and were confirmed using complex wholebody sports skills. Temporal occlusion was also consistently created where it was intended, relative to the kinematic events of the sports skills. The laser curtain presents promising opportunities for use in visual-perception-action research in sports and other disciplines.

\section{References}

Abernethy, B., Gill, D. P., Parks, S. L., \& Packer, S. T. (2001). Expertise and the perception of kinematic and situational probability information. Perception, 30, 233-252. doi:10. 1068/p2872

Burroughs, W. A. (1984). Visual simulation training of baseball batters. International Journal of Sport Psychology, 15, 117-126.

Farrow, D., \& Abernethy, B. (2002). Can anticipatory skills be learned through implicit video-based perceptual training? Journal of Sports Sciences, 20, 471-485. doi:10.1080/02640410252925143

Farrow, D., \& Abernethy, B. (2003). Do expertise and the degree of perception-action coupling affect natural anticipatory performance? Perception, 32, 1127-1139. doi:10.1068/p3323

Farrow, D., Abernethy, B., \& Jackson, R. C. (2005). Probing expert anticipation with the temporal occlusion paradigm: Experimental investigations of some methodological issues. Motor Control, 9, 332-351.

Loffing, F., \& Hagemann, N. (2014). Skill differences in visual anticipation of type of throw in team-handball penalties. Psychology of Sport and Exercise, 15, 260-267. doi:10.1016/j.psychsport.2014.01.006

Mann, D. L., Abernethy, B., Farrow, D., Davis, M., \& Spratford, W. (2010). An event-related visual occlusion method for examining anticipatory skill in natural interceptive tasks. Behavior Research Methods, 42, 556-562. doi:10.3758/BRM.42.2.556

Mann, D. L., Abernethy, B., \& Farrow, D. (2010). Action specificity increases anticipatory performance and the expert advantage in natural interceptive tasks. Acta Psychologica, 135, 17-23. doi:10.1016/j.actpsy.2010.04.006

Marinovic, W., Plooy, A. M., \& Tresilian, J. R. (2009). The utilisation of visual information in the control of rapid interceptive actions. Experimental Psychology, 56, 265-273. doi:10.1027/1618-3169.56.4.265

Milgram, P. (1987). A spectacle-mounted liquid-crystal tachistoscope. Behavior Research Methods, Instruments, \& Computers, 19, 449456. doi:10.3758/BF03205613

Morris, S. L., Foster, C. J., Parsons, R., Falkmer, M., Falkmer, T., \& Rosalie, S. M. (2015). Differences in the use of vision and proprioception for postural control in autism spectrum disorder. Neuroscience, 307, 273280. doi:10.1016/j.neuroscience.2015.08.040

Müller, S., \& Abernethy, B. (2006). Batting with occluded vision: An in situ examination of the information pick-up and interceptive skills of high- and low-skilled cricket batsmen. Journal of Science and Medicine in Sport, 9, 446-458. doi:10.1016/j.jsams.2006.03.029

Müller, S., Abernethy, B., Reece, J., Rose, M., Eid, M., McBean, R., ... Abreu, C. (2009). An in-situ examination of the timing of information pick-up for interception by cricket batsmen of different skill levels. Psychology of Sport and Exercise, 10, 644652. doi:10.1016/j.psychsport.2009.04.002

Müller, S., Abernethy, B., \& Farrow, D. (2006). How do worldclass cricket batsmen anticipate a bowler's intention? 
Quarterly Journal of Experimental Psychology, 59, 21622186. doi:10.1080/02643290600576595

Müller, S., Brenton, J., Dempsey, A. R., Harbaugh, A. G., \& Reid, C. (2015). Individual differences in highly skilled visual perceptualmotor striking skill. Attention, Perception \& Psychophysics, 77, 1726-1736. doi:10.3758/s13414-015-0876-7

Müller, S., Lalović, A., Dempsey, A. R., Rosalie, S., \& Harbaugh, A. G. (2014). Pick-up of early visual information to guide kinetics and kinematics within a group of highly skilled baseball batters. Perceptual and Motor Skills, 119, 347-362. doi:10.2466/30.PMS.119c21z9

Starkes, J. L., Edwards, P., Dissanayake, P., \& Dunn, T. (1995). A new technology and field test of advance cue usage in volleyball.
Research Quarterly for Exercise and Sport, 66, 162-167. doi: 10.1080/02701367.1995.10762223

van Soest, A. J. K., Casius, L. J. R., de Kok, W., Krijger, M., Meeder, M., \& Beek, P. J. (2010). Are fast interceptive actions continuously guided by vision? Revisiting Bootsma and van Wieringen (1990). Journal of Experimental Psychology: Human Perception and Performance, 36, 1040-1055. doi:10.1037/a0016890

Whitwell, R. L., Lambert, L. M., \& Goodale, M. A. (2008). Grasping future events: Explicit knowledge of the availability of visual feedback fails to reliably influence prehension. Experimental Brain Research, 188, 603-611. doi:10.1007/s00221-008-1395-8 